\title{
Abbreviated San Diego Wisdom Scale (SD-WISE-7) and Jeste-Thomas Wisdom Index (JTWI)
}

\author{
Michael L. Thomas, ${ }^{1}$ (D) Barton W. Palmer, 2,3,4 (D) Ellen E. Lee, 2,3,4 Jinyuan Liu, ${ }^{4,5}$ \\ Rebecca Daly, ${ }^{2,4}$ Xin M. Tu, ${ }^{4,5}$ and Dilip V. Jeste 2,4,6 \\ ${ }^{1}$ Department of Psychology, Colorado State University, Fort Collins, CO, USA \\ ${ }^{2}$ Department of Psychiatry, University of California San Diego, La folla, CA, USA \\ ${ }^{3}$ VA San Diego Healthcare System, San Diego, CA, USA \\ ${ }^{4}$ Sam and Rose Stein Institute for Research on Aging, University of California San Diego, La folla, CA, USA \\ ${ }^{5}$ Department of Family Medicine and Public Health, University of California San Diego, La folla, CA, USA \\ ${ }^{6}$ Department of Neurosciences, University of California San Diego, La folla, CA, USA
}

Objectives: Wisdom is a personality trait comprising seven components: self-reflection, pro-social behaviors, emotional regulation, acceptance of diverse perspectives, decisiveness, social advising, and spirituality. Wisdom, a potentially modifiable trait, is strongly associated with well-being. We have published a validated 28-item San Diego Wisdom Scale, the SD-WISE-28. Brief scales are necessary for use in large populationbased studies and in clinical practice. The present study aimed to create an abbreviated 7-item version of the SD-WISE.

Method: Participants included 2093 people, aged 20-82 years, recruited and surveyed through the online crowdsourcing platform Amazon Mechanical Turk. The participants' mean age was 46 years, with 55\% women. Participants completed the SD-WISE-28 as well as validation scales for various positive and negative constructs. Psychometric analyses (factor analysis and item response theory) were used to select one item from each of the seven SD-WISE-28 subscales.

Results: We selected a combination of items that produced acceptable unidimensional model fit and good reliability $(\omega=0.74)$. Item statistics suggested that all seven items were strong indicators of wisdom, although the association was weakest for spirituality. Analyses indicated that the 28 -item and 7 -item SD-WISE are both very highly correlated $(\mathrm{r}=0.92)$ and produce a nearly identical pattern of correlations with demographic and validity variables.

Conclusion: The SD-WISE-7, and its derived Jeste-Thomas Wisdom Index (JTWI) score, balances reliability and brevity for research applications.

Key words Compassion, Spirituality, Loneliness, Well-being, Depression, Aging

\section{Introduction}

What is wisdom and how is it measured? Despite the long history of religious and philosophical literature on wisdom, empirical research in this area has been a relatively recent phenomenon, starting in the 1970s. However, the scientific publications on wisdom have been increasing rapidly, especially during the last decade (Jeste and Lee, 2019). A special issue of a major journal, Psychological Inquiry, on Wisdom

Correspondence should be addressed to: Dilip V. Jeste, Sam and Rose Stein Institute for Research on Aging, University of California San Diego, 9500 Gilman Drive, La Jolla, CA 92093-0664, USA. Email: djeste@ucsd.edu Received 02 Jul 2021; revised version received 01 Oct 2021; accepted 20 Oct 2021. First published online 03 December 2021. was published in 2020 (Grossmann et al., 2020; Jeste et al., 2020a). Wisdom measures are increasingly being used to study factors that promote mental health and optimal aging, and have been shown to be associated with a variety of positive outcomes such as happiness, mental and physical health, and self-rated successful aging (Ardelt and Jeste, 2016; Ardelt, 1997; Etezadi and Pushkar, 2013; Grossmann et al., 2020; Jeste and Lee, 2019). Notwithstanding the progress in the research on wisdom, measures of wisdom have not yet found wide usage in large cross-sectional and longitudinal studies. One possible reason has been a lack of brief but validated measures of wisdom that can be used in regular research or clinical investigations. Measures of wisdom typically include a relatively large number 
of items (i.e., 28-40 items) (Ardelt, 2003; Thomas et al., 2019a; Webster, 2003), which can consume precious time and effort in large-scale research studies. In recent decades, in nearly all domains of measurement, there has been a growing trend towards developing short but reliable measures that can be more easily worked into a study's assessment battery (Cella et al., 2019)

Wisdom is a complex, multi-component personality trait (Bangen et al., 2013). Our group created the 24-item San Diego Wisdom scale (Thomas et al., 2019a) that assesses six components of wisdom. The self-reflection component measures the desire and ability to understand oneself and one's actions at a deeper level. It assesses preferences with regard to understanding one's own thoughts, motivations, and behaviors. The pro-social behaviors component includes empathy, compassion, altruism, and sense of fairness. It evaluates one's sense of the ability to maintain positive social connections, as well as compassion or conscientious behavior. The emotional regulation component measures the ability to regulate negative emotions that interfere with decision making. It assesses one's sense of being able to effectively manage negative emotions and emotional stress and to favor positive feelings. The acceptance of divergent perspectives component examines acceptance of other value systems and interest in learning others' perspectives. It measures one's openness to and comfort with values and perspectives that may be different from one's own values and perspectives. The decisiveness component evaluates the ability to make decisions in a timely manner. It also assesses one's comfort with decision making. The social advising component refers to the ability to give good advice to others.

Subsequently, because of an increasing number of publications suggesting that spirituality is also a component of wisdom, we added this new component to the SD-WISE (Jeste et al., 2021). Although a newer component of the SD-WISE, spirituality has been considered to be a component of wisdom for centuries. It was an integral part of wisdom in religious scriptures (Achenbaum and Orwoll, 1991; Jeste and Vahia, 2008). In a review of the modern empirical literature on wisdom (Bangen et al., 2013), we found a number of reports that included spirituality in the definition of wisdom (Hershey and Farrell, 1997; Jason et al., 2001; Perry et al., 2002; Wink and Helson, 1997). Several other studies have reported a significant correlation between spirituality and greater well-being (Koenig, 2012; de Jager Meezenbroek et al., 2012), similar to the association between wisdom and mental health discussed earlier in this article. The spirituality component measures connectedness with oneself, with the nature, or with the transcendent like the soul or God. We labeled the total score on this expanded 28-item scale the Jeste-Thomas Wisdom Index or JTWI.

In previous work, we have shown that scores on the original SD-WISE are reliable, valid, and have positive correlations with measures of good mental health and well-being as well as negative correlations with measures of poor mental health (Jeste et al., 2021). The scale is increasingly being used in large national and international studies (Jeste et al., 2020a, 2020b; Nguyen et al., 2020), biological research (Grennan et al., 2021; Nguyen et al., 2021; Thomas et al., 2019b), and clinical trials (Treichler et al., 2020), including a recent study that demonstrated positive change in specific components of the SD-WISE following a mindfulness intervention (Al-Refae et al., 2021).

The SD-WISE is modestly correlated with two other measures of wisdom that have been reported to have good psychometric properties: Ardelt's Three Dimensional Wisdom Scale (3D-WS; Ardelt, 2003) and Webster's Self-Assessed Wisdom Scale (SAWS; Webster, 2003). The 3D-WS is a 39-item measure, and the SAWS is a 40 -item measure. Thus, the SD-WISE, 3D-WS, and SAWS are all quite long relative to many commonly used outcome measures. Our group, in collaboration with Ardelt, recently developed a shortened 12-item version of the 3D-WS (Thomas et al., 2017). A similar effort reduced the SAWS to 15 items (Leeman et al., 2021). However, the 3D-WS, SAWS, and SDWISE differ in several important ways. Most notably, the scales assess different subcomponents of wisdom and are based on different theoretical models. Total scores from the measures - while moderately correlated - share less than $25 \%$ of variance in common (Thomas et al., 2019a). As such, 3D-WS, SAWS, and SD-WISE scores are not interchangeable. Moreover, the scales have not been psychometrically linked. Thus, given the increasing popularity of the SD-WISE, there is a need for an abbreviated version.

The current study aimed to develop a reliable and valid abbreviated version of SD-WISE with only one item for each of the seven components mentioned above. A 7-item wisdom measure would be comparable to many existing short-form outcome measures relevant to health, such as the fixed-item NIH Patient-Reported Outcomes Measurement Information System (PROMIS) measures (Cella et al., 2007).

\section{Methods}

\section{Sample and Design}

Participants included 2093 people, aged 20-82 years, recruited and surveyed through the online 
crowdsourcing platform Amazon Mechanical Turk (MTurk-AMT). MTurk-AMT has been used in a number of large cross-sectional studies of various health measures (Buhrmester et al., 2011; Litman et al., 2015; Mason and Suri, 2012; Nguyen et al., 2020; Sprouse, 2011). Our Inclusion criteria were: 1) Age $\geq 20$ years, 2) English-speaking, 3) resident of the United States, and 4) MTurk - AMT Human Intelligence Task (HIT) approval rating $\geq 90 \%$, indicating that the respondent's previous HITs had been approved by requestors at least $90 \%$ of the time. AMT has been shown to produce high quality data; however, a small proportion of responses could be invalid due to inattentiveness or other reasons. Therefore, to further ensure data validity, we applied a data cleaning procedure to help identify and remove participants who provided impossible or highly implausible responses to specific survey questions, consistent with the methods employed in a number of published AMT studies by various investigators (Coppock, 2019; Hauser and Schwarz, 2016; Nguyen et al., 2020; Peer et al., 2014). Specifically, we excluded participants who 1) completed the survey in $<390$ seconds $(\mathrm{N}=297)$, 2) reported a height and weight resulting in a BMI $<$ $16(\mathrm{~N}=12), 3)$ reported their height at $<3$ feet or $>7$ feet $(n=3)$, or 4$)$ reported living with $\geq 20$ people in their household $(n=3)$. Please note that these were not a priori exclusion criteria, but were based on post-hoc finding of a small minority of surveys that included responses that were far beyond the reported range in this population, and therefore, appeared to be extremely unlikely to be valid, per other participants' responses. Thus, data were excluded from a total of 307 respondents, resulting in a final sample of 1786 participants whose data were included in the current analysis.

The participants' mean age was 46.3 years, with SD $14.6 ; 55 \%$ were women; $77 \%$ were Caucasian, 9\% Hispanic/Latinx, 7\% African American, 4\% Asian American, and 3\% belonged to another race/ethnicity. In terms of education, $44 \%$ subjects had a high school diploma, $41 \%$ had a bachelor's degree, and $14 \%$ had masters or doctorate. About half $(51 \%)$ were married or cohabitating.

The study was approved by the UCSD Office of IRB Administration (OIA) (also called Human Research Protections Program); with a waiver of signed consent under the provisions of $45 \mathrm{CFR}$ 46.104(d), Category 2.

\section{Measures}

As described above, we used the 28-item SD-WISE with all the seven components of wisdom. Each component includes four items. The items are rated from 1 or "strongly disagree" to 5 or "strongly agree".
Negatively worded items are reverse scored. The seven component scores are then calculated by averaging the four included items, and an overall wisdom score (JTWI) is calculated by averaging all 28 items.

Convergent validity measures included the twoitem Patient Health Questionnaire Depression Module (PHQ-2; Kroenke et al., 2001), the 12-Item Medical Outcomes Study Short Form Health Survey - Physical and Mental Components (MOS-12; Ware and Sherbourne, 1992), the two-item ConnorDavidson Resilience Scale (CD-RISC; CampbellSills and Stein, 2007), the Center for Epidemiologic Studies Depression Scale Happiness Factor (CESDHS; Fowler and Christakis, 2008), the two-item Generalized Anxiety Disorder Scale (GAD-2; Kroenke et al., 2007), the four-item version of the UCLA Loneliness Scale (UCLA-4) for loneliness (UCLA-4; Russell et al., 1978), and the Duke Social Interaction Subscale (DSSI; Koenig et al., 1993). We expected positive correlations with MOS-12, CDRISC, CESD-HS, and DSSI, and negative correlations with PHQ-2, GAD-2, and UCLA-4.

\section{Analyses}

Our goal was to create a 7 -item version of the SDWISE (i.e., SD-WISE-7), with one item for each component of wisdom. Several planned psychometric analyses were used to select the final 7-item scale. To begin, we evaluated the item properties of all 28 SD-WISE items in order to ensure good psychometric properties. Specifically, in order to evaluate item properties, we fitted item response theory (IRT) graded response models to the data. Discrimination parameters indicate the strength of association between the latent trait measured by a scale and endorsement of the response options. Higher discrimination values are preferred. Threshold parameters indicate the extent to which higher levels of a trait are required to endorse higher item response options. For ordered categorical response data, widely spaced and balanced (i.e., both positive and negative) threshold parameters are preferred. Parameters were estimated using the $\mathrm{R}$ ltm package (Rizopoulos, 2006). Reverse-coded items were rescored prior to analyses. Next, in order to evaluate dimensionality, we fitted unidimensional confirmatory factor models to all possible seven-item combinations of SD-WISE items, always drawing one item from each of the seven subscales to ensure balanced content. Models were fitted to the data using the $\mathrm{R}$ lavaan package (Rosseel, 2012) with full information maximum likelihood estimation and robust standard errors (MLR). (Both robust maximum likelihood and diagonally weighted least squares estimation can be used for categorical data. Although results are generally comparable, both 
have strengths and weaknesses (Li, 2016)). Model fit was based on the comparative fit index (CFI), Tucker-Lewis index (TLI), and root mean square error of approximation (RMSEA) statistics. CFI and TLI values of approximately 0.95 or greater and RMSEA values of approximately 0.06 and lower are typically considered excellent; CFI and TLI values above 0.90 and RMSEA values below 0.08 are considered adequate (Brown, 2015; Hu and Bentler, 1999). Next, we used IRT to examine the psychometric properties of the newly created 7-item SD-WISE. Reliability coefficient omega $(\omega)$ was estimated using the R psych package (Revelle, 2018). Although coefficient alpha has more commonly been used to estimate reliability, McDonald's omega makes fewer assumptions about the data, and therefore provides a more accurate index of reliability (McDonald, 1999). In particular, omega corrects for underestimation of reliability and is therefore preferred by methodologists (Hayes and Coutts, 2020). In general, reliability values of 0.7 or greater are considered acceptable (Haynes et al., 2011). In order to evaluate the validity of the SD-WISE-7, we correlated 7-item total scores (JTWI) with the original 28-item SD-WISE scores, as well as demographic and mental health variables.

\section{Results}

Item discrimination parameter estimates for all 28items of the SD-WISE-28 are reported in Table 1. Notably, all of the discrimination parameters are positive, and most have modest to large effect sizes, suggesting that the items are good indicators of their measured constructs. Only one item - item 14 from the Acceptance of Divergent Perspectives subscale falls below one. The threshold parameters are generally well-spaced, suggesting broad coverage of trait levels within each subscale. In general, the item parameter estimates did not indicate that any item should be excluded from inclusion in the SDWISE-7.

Model fit statistics for the best five combinations of items are reported in Table 2. In terms of goodness-of-fit overall, all five models had excellent to good RMSEA values and adequate CFI values. TLI values are slightly below the border of acceptable fit, but close enough to the approximate value to be considered marginally acceptable (particularly for the best fitting item combination). As can be seen, model fit statistics were very similar between these top five solutions, meaning that any of these combinations of items would perform adequately. Moreover, all combinations included item 11 ("I tend to postpone making major decisions as long as I can.” Reverse-coded) for the Decisiveness subscale, item 16
("I avoid self-reflection." Reverse-coded) for SelfReflection, item 5 ("I avoid situations where I know my help will be needed." Reverse-coded) for Pro-Social Behaviors, and item 23 ("I often don't know what to tell people when they come to me for advice." Reverse-coded) for Social Advising, suggesting that these items were consistently optimal indicators of the constructs assessed. For emotional regulation, the choice was between item 15 ("I am able to recover well from emotional stress.") and item 9 ("I remain calm under pressure."). Ultimately, we chose item 9 because it had a larger subscale discrimination parameter, had a shorter word length, and was judged by the authors to have better content validity (i.e., calm response as opposed to emotional recovery). For Acceptance of Divergent Perspectives, the choice was between item 21 ("I enjoy being exposed to diverse viewpoints.") and item 10 ("I enjoy learning things about other cultures."). We chose item 21 because it had a larger subscale discrimination parameter and was judged to have better content validity (i.e., explicit focus on diverse ideas). Finally, for spirituality, the choice was between items 5 ("My spiritual belief gives me inner strength.") and 3 ("There is no existence of the soul after death."). We chose item 5 because it had a larger subscale discrimination parameter, had a shorter word length, and was judged to have better content validity (i.e., a greater focus on belief rather than on religious archetypes).

Item discrimination parameter estimates for the SD-WISE-7 are reported in Table 3. As with subscale item analyses, the threshold parameters are generally well-spaced, suggesting broad coverage of trait levels (i.e., people at mostly all levels of wisdom are expected to be measured with similar precision). The largest discrimination parameters were for "I often don't know what to tell people when they come to me for advice", "I avoid situations where I know my help will be needed", and "I tend to postpone making major decisions as long as I can", indicators of social advising, pro-social behavior, and decisiveness, respectively. More modest discrimination parameters were produced for "I remain calm under pressure", "I avoid self-reflection", and "I enjoy being exposed to diverse viewpoints", indicators of emotional regulation, self-reflection, and acceptance of divergent perspectives, respectively. The smallest discrimination parameter was for the spirituality item, "My spiritual belief gives me inner strength".

Reliability coefficients for the 28 - and 7-item SDWISE scale scores were $\omega=0.84$ and 0.74 , respectively. Thus, while the 28 -item SD-WISE produced better reliability, the 7-item SD-WISE is still acceptably reliable by conventional standards. 
Table 1. Item discrimination parameter estimates for the SD-WISE subscales

\begin{tabular}{|c|c|c|c|c|c|}
\hline \multirow[b]{2}{*}{ ITEM } & \multirow[b]{2}{*}{ DISCRIMINATION } & \multicolumn{4}{|c|}{ THRESHOLDS } \\
\hline & & 1 & 2 & 3 & 4 \\
\hline \multicolumn{6}{|l|}{ Decisiveness } \\
\hline SDWISE 2: I have trouble making decisions. (R) & 3.20 & -1.69 & -2.55 & -1.74 & -2.51 \\
\hline SDWISE 8: I usually make decisions in a timely fashion. & 2.44 & -0.81 & -1.36 & -0.77 & -1.25 \\
\hline SDWISE 11: I tend to postpone making major decisions as long as I can. (R) & 2.69 & -0.43 & -0.73 & -0.30 & -0.50 \\
\hline $\begin{array}{l}\text { SDWISE 17: I would rather someone else make the decision for me if I am } \\
\text { uncertain. (R) }\end{array}$ & 1.48 & 0.89 & 1.04 & 0.87 & 0.91 \\
\hline \multicolumn{6}{|l|}{ Emotional regulation } \\
\hline SDWISE 6: I have trouble thinking clearly when I am upset. (R) & 2.04 & -1.68 & -2.06 & -2.23 & -2.44 \\
\hline SDWISE 9: I remain calm under pressure. & 2.76 & -0.42 & -1.14 & -1.14 & -1.33 \\
\hline SDWISE 15: I am able to recover well from emotional stress. & 2.53 & 0.14 & -0.51 & -0.45 & -0.59 \\
\hline SDWISE 24: I cannot filter my negative emotions. (R) & 1.79 & 1.34 & 0.92 & 1.21 & 0.95 \\
\hline \multicolumn{6}{|l|}{ Self-reflection } \\
\hline SDWISE 12: I take time to reflect on my thoughts. & 1.68 & -3.36 & -2.38 & -3.92 & -2.3 \\
\hline SDWISE 16: I avoid self-reflection. (R) & 2.82 & -2.34 & -1.55 & -2.53 & -1.51 \\
\hline SDWISE 19: It is important that I understand the reasons for my actions. & 1.30 & -1.24 & -1.03 & -1.52 & -1.01 \\
\hline SDWISE 22: I don't analyze my own behavior. (R) & 2.65 & 0.85 & 0.44 & 0.97 & 0.53 \\
\hline \multicolumn{6}{|l|}{ Pro-social behaviors } \\
\hline SDWISE 3: I have a difficult time keeping friendships. (R) & 1.37 & -2.51 & -2.74 & -3.97 & -4.21 \\
\hline SDWISE 5: I avoid situations where I know my help will be needed. (R) & 2.12 & -1.31 & -1.66 & -3.00 & -3.10 \\
\hline $\begin{array}{l}\text { SDWISE 13: I would stop a stranger who dropped a twenty-dollar bill to } \\
\text { return it. (R) }\end{array}$ & 1.18 & -0.67 & -0.99 & -2.19 & -2.17 \\
\hline $\begin{array}{l}\text { SDWISE 18: I treat others the way I would like to be treated. } \\
\text { Social advising }\end{array}$ & 1.46 & 0.98 & 0.6 & -0.05 & 0.07 \\
\hline SDWISE 1: I am good at perceiving how others are feeling. & 1.24 & -4.57 & -2.48 & -2.44 & -3.44 \\
\hline SDWISE 4: Others look to me to help them make choices. & 1.84 & -2.53 & -1.20 & -1.63 & -1.81 \\
\hline SDWISE 7: Others say I give good advice. & 3.29 & -1.56 & -0.16 & -0.61 & -0.70 \\
\hline $\begin{array}{l}\text { SDWISE 23: I often don't know what to tell people when they come to me for } \\
\text { advice. (R) }\end{array}$ & 1.29 & 1.58 & 2.03 & 1.07 & 1.26 \\
\hline \multicolumn{6}{|l|}{ Acceptance of divergent perspectives } \\
\hline SDWISE 10: I enjoy learning things about other cultures. & 1.59 & -3.22 & -5.45 & -3.83 & -2.27 \\
\hline $\begin{array}{l}\text { SDWISE 14: I am okay with others having morals and values other than } \\
\text { my own. }\end{array}$ & 0.89 & -2.16 & -3.53 & -1.95 & -1.52 \\
\hline SDWISE 20: I generally learn something from every person I meet. & 1.10 & -1.26 & -1.71 & -0.81 & -0.73 \\
\hline $\begin{array}{l}\text { SDWISE 21: I enjoy being exposed to diverse viewpoints. } \\
\text { Spirituality }\end{array}$ & 3.79 & 0.44 & 1.52 & 1.74 & 0.74 \\
\hline SDWISE 3: There is no existence of the soul after death. (R) & 3.06 & -1.19 & -0.77 & -1.31 & -2.11 \\
\hline SDWISE 5: My spiritual belief gives me inner strength. & 3.68 & -0.74 & -0.36 & -0.81 & -1.40 \\
\hline SDWISE 10: I feel that we are all connected on a higher level. & 2.62 & -0.07 & 0.05 & -0.05 & -0.70 \\
\hline SDWISE 13: There is no overall purpose to life. (R) & 1.81 & 0.47 & 0.85 & 0.95 & 0.11 \\
\hline
\end{tabular}

The italicized items were ultimately selected. $(\mathrm{R})=$ reversed coded.

Concurrent validity, demographic, and construct validity correlations for the 28 - and 7 -item versions of the SD-WISE are reported in Table 4. The correlation between 28- and 7-item SD-WISE total scores was 0.92 , indicating that the reduced-length scale is an excellent indicator of the original measure. Moreover, the patterns of correlations between 28- and 7-item SD-WISE total scores and both demographic and validity variables were largely similar. Both measures were positively correlated with age, negatively correlated with male sex, and, weakly, but positively correlated with education. Additionally, both measures were negatively correlated with loneliness (UCLA-4), depression (PHQ-2), and anxiety (GAD-2), and positively correlated with mental well-being (MOS-12 Mental), resilience (CD-RISC), happiness (CESD-HS), and social interaction (DSSI). Both were very weakly correlated with physical well-being (MOS-12 Physical).

\section{Discussion}

We aimed to develop and test a shortened, 7-item version of the 7 -subscale 28 -item SD-WISE. Using 
Table 2. Unidimensional model fit indices for the top five combinations of items

\begin{tabular}{|c|c|c|c|}
\hline ITEM COMBINATION & $\begin{array}{l}\text { COMPARATIVE FIT } \\
\text { INDEX (CFI) }\end{array}$ & $\begin{array}{l}\text { TUCKER LEWIS } \\
\text { INDEX (TLI) }\end{array}$ & $\begin{array}{l}\text { ROOT MEAN SQUARE ERROR OF } \\
\text { APPROXIMATION (RMSEA) }\end{array}$ \\
\hline $\begin{array}{l}D: 11, E R: 9, S R: 16, P S B: 5, S A: \\
\quad 23, T D V: 21, S: 5\end{array}$ & 0.92 & 0.88 & 0.06 \\
\hline $\begin{array}{l}\text { D: } 11 \text {, ER: } 15 \text {, SR: } 16 \text {, PSB: } 5 \text {, SA: } \\
23 \text {, TDV: } 21, \text { S: } 3\end{array}$ & 0.91 & 0.87 & 0.06 \\
\hline $\begin{array}{l}\text { D: } 11 \text {, ER: } 15 \text {, SR: } 16 \text {, PSB: } 5 \text {, SA: } \\
\text { 23, TDV: } 21 \text {, S: } 5\end{array}$ & 0.91 & 0.86 & 0.07 \\
\hline $\begin{array}{l}\text { D: } 11 \text {, ER: } 9 \text {, SR: } 16 \text {, PSB: } 5 \text {, SA: } \\
\text { 23, TDV: } 21, \text { S: } 3\end{array}$ & 0.90 & 0.86 & 0.07 \\
\hline $\begin{array}{l}\text { D: } 11 \text {, ER: } 15 \text {, SR: } 16 \text {, PSB: } 5 \text {, SA: } \\
23 \text {, TDV: } 10, \text { S: } 3\end{array}$ & 0.90 & 0.86 & 0.07 \\
\hline
\end{tabular}

$\mathrm{D}$ = Decisiveness; ER = Emotional Regulation; SR = Self-Reflection; PSB = Pro-Social Behaviors; SA = Social Advising; TDV = Tolerance for Divergent Values; $S=$ Spirituality. The italicized combination of items was ultimately selected.

Table 3. Item discrimination parameter estimates for the seven-item SD-WISE

\begin{tabular}{|c|c|c|c|c|c|}
\hline \multirow[b]{2}{*}{ ITEM } & \multirow[b]{2}{*}{ DISCRIMINATION } & \multicolumn{4}{|c|}{ THRESHOLDS } \\
\hline & & 1 & 2 & 3 & 4 \\
\hline SD-WISE7 1: I remain calm under pressure. & 1.08 & -3.33 & -1.82 & -0.84 & 1.45 \\
\hline SD-WISE7 2: I avoid self-reflection. (R) & 1.03 & -4.18 & -2.57 & -1.64 & 0.76 \\
\hline SD-WISE7 3: I enjoy being exposed to diverse viewpoints. & 0.80 & -5.30 & -3.33 & -1.54 & 1.56 \\
\hline $\begin{array}{l}\text { SD-WISE7 4: I tend to postpone making major decisions } \\
\text { as long as I can. (R) }\end{array}$ & 1.52 & -2.20 & -0.99 & -0.42 & 1.10 \\
\hline $\begin{array}{l}\text { SD-WISE7 5: I often don't know what to tell people when they come to me } \\
\text { for advice. (R) }\end{array}$ & 1.76 & -2.80 & -1.50 & -0.60 & 1.07 \\
\hline SD-WISE7 6: My spiritual belief gives me inner strength. & 0.33 & -3.61 & -1.71 & 0.12 & 3.90 \\
\hline SD-WISE7 7: I avoid situations where I know my help will be needed. (R) & 1.92 & -2.88 & -1.72 & -1.01 & 0.62 \\
\hline
\end{tabular}

The italicized items were ultimately selected. $(\mathrm{R})=$ reversed coded.

Table 4. Concurrent validity, demographic, convergent validity correlations

\begin{tabular}{|c|c|c|c|c|c|c|}
\hline & \multicolumn{3}{|c|}{ SD-WISE-28 } & \multicolumn{3}{|c|}{ SD-WISE-7 } \\
\hline & $\mathrm{CI}_{5 \%}$ & $r$ & $\mathrm{CI}_{95 \%}$ & $\mathrm{CI}_{5 \%}$ & $r$ & $\mathrm{CI}_{95 \%}$ \\
\hline Wisdom (SD-WISE-28) & & - & & 0.91 & 0.92 & 0.93 \\
\hline Age & 0.17 & 0.22 & 0.26 & 0.16 & 0.21 & 0.25 \\
\hline Sex (Male) & -0.18 & -0.14 & -0.09 & -0.15 & -0.10 & -0.06 \\
\hline Education & 0.00 & 0.04 & 0.09 & 0.01 & 0.06 & 0.1 \\
\hline Loneliness (UCLA-4) & -0.56 & -0.53 & -0.5 & -0.5 & -0.47 & -0.43 \\
\hline Mental well-being (MOS-12) & 0.50 & 0.53 & 0.57 & 0.46 & 0.49 & 0.53 \\
\hline Physical well-being (MOS-12) & 0.01 & 0.06 & 0.1 & 0.01 & 0.06 & 0.10 \\
\hline Depression (PHQ-2) & -0.52 & -0.49 & -0.45 & -0.48 & -0.45 & -0.41 \\
\hline Anxiety (GAD-2) & -0.48 & -0.45 & -0.41 & -0.46 & -0.42 & -0.38 \\
\hline Resilience (CD-RISC) & 0.57 & 0.60 & 0.63 & 0.52 & 0.56 & 0.59 \\
\hline Happiness (CESD-HS) & 0.53 & 0.56 & 0.60 & 0.47 & 0.51 & 0.54 \\
\hline Social Interaction (DSSI) & 0.28 & 0.32 & 0.37 & 0.23 & 0.28 & 0.32 \\
\hline
\end{tabular}

$\mathrm{CI}_{5 \%}=$ lower 5\% confidence interval; $\mathrm{CI}_{95 \%}=$ upper 95\% confidence interval. CD-RISC = Connor-Davidson Resilience Scale; CESDHS = Center for Epidemiological Studies Depression Scale Happiness Factor; DSSI = Duke Social Support Index; GAD-2 = 2-item Generalized Anxiety Disorder Scale; MOS-12 = Medical Outcomes Survey Short Form; PHQ-2 = 2-item Patient Health Questionnaire; SDWISE-28 = 28-item San Diego Wisdom Scale; UCLA-4 = 4-item UCLA Loneliness Scale. 
several psychometric analysis techniques, we selected one item from each of the seven SDWISE subscales. The selected combination of items produced acceptable unidimensional model fit. Thus, the new 7-item version of the SD-WISE the SD-WISE-7 - measures a single latent factor the putative Wisdom factor underlying the JTWI. Item statistics for the SD-WISE-7 suggested that most items were strong indicators of wisdom. A notable exception was the spirituality item, which produced a more modest discrimination parameter. In fact, this finding in similar to our previous work that also found spirituality to be a relatively weak, although significant, indicator of the total score on the 28-item SD-WISE (Jeste et al., 2021)

Reliability analysis for the SD-WISE-7 was $\omega=0.74$ which is generally considered acceptable for research purposes, but should be acceptably reliable (Haynes et al., 2011). Statistical power for detecting significant effects should be reasonable for moderate to large samples (but ultimately depends on effect size). However, studies employing small samples may wish to use the 28-item SD-WISE, which, as would be expected, produces more reliable scores.

Demographic, construct validity, and concurrent validity correlations indicated that the 28 - and 7item SD-WISE are both very highly correlated and produce a nearly identical pattern of correlations with demographic and validity variables. Thus, the results observed using the 28- and 7-item SD-WISE total scores (JTWIs) should be comparable. In particular, as with the SD-WISE-28, the results suggest that the SD-WISE-7 is most strongly and positively correlated with resilience, happiness, and mental well-being, as well as most strongly and negatively correlated with loneliness, depression, and anxiety. This pattern is broadly consistent with our previous findings that wisdom is associated with a variety of positive traits and outcomes (Ardelt and Jeste, 2016; Ardelt, 1997; Etezadi and Pushkar, 2013; Grossmann et al., 2020; Jeste and Lee, 2019).

Despite this, because lower reliability implies greater error and smaller effect size, associations between SD-WISE-7 scores and other variables should generally be smaller than associations between SD-WISE-28 scores and other variables. In fact, this phenomenon is shown in Table 4, where the SD-WISE-7 validity correlations are consistently smaller (albeit slightly) than SD-WISE-28 validity correlations.

Although response times were not available for the current data, our past research suggests that SDWISE items take an average of 5.5 seconds to complete. Thus, the SD-WISE-7 is expected to have an administration time of about one minute, and should be approximately $2-3$ minutes faster to complete in comparison to the SD-WISE-28.
Several studies have reported an association of wisdom with aging, although much of this literature is based on cross-sectional research. Thus, the current study's findings of positive association of JTWI with aging, female sex, and with positive characteristics like mental well-being, resilience, happiness, and social interaction, and negative association with depression and anxiety are consistent with much of the published research on wisdom (Ardelt and Jeste, 2016; Ardelt, 1997; Etezadi and Pushkar, 2013; Grossmann et al., 2020; Jeste and Lee, 2019). Particularly notable is the relationship with loneliness. Several US-based studies have reported a significant inverse correlation between SD-WISE total scores (JTWI) and loneliness (Jeste et al., 2021; Lee et al., 2019; Nguyen et al., 2020). Another study with a different sample from our research group (Jeste et al., 2020b) compared older adults from the USA with those from the Cilento region of Italy using English and Italian versions, respectively, of these scales. The study found that the basic constructs of wisdom and loneliness seemed to be similar in the two cultures, with a consistent inverse correlation between them.

Two recent biological investigations using SDWISE and loneliness also showed inverse biological correlates of these entities. An EEG study found that wisdom was related to enhanced response speed biased by happy emotions whereas loneliness was associated with reduced response speed biased by angry emotions, and both invoked similar neural circuits (i.e., temporo-parietal junction) (Grennan et al., 2021). A study of gut microbiome found that wisdom and loneliness were associated with higher versus lower, respectively, levels of alpha and beta diversity, which are known to be markers of better versus worse health, respectively (Nguyen et al., 2020).

This study has several strengths including a relatively large size of the sample that included adults across the lifespan and use of multiple validity measures. Nonetheless, it does have some limitations. The study sample was predominantly comprised of people from non-Latinx white race/ethnicity and high levels of education relative to the general US public. Thus, the findings may not generalize to people from diverse socioeconomic or other racial/ ethnic groups. All of the measures used were based on self-report and thus associations could be affected by response biases such as social desirability and/or response patterns (e.g. tendency to use or avoid extreme points in ratings, tendency to rate all scales in the "negative" or "positive" direction, etc.). However, in previous research, we have found that social desirability bias does not play a substantial role in self-reports of well-being and related measures (Dawes et al., 2011). While objective measures 
would be helpful, there are currently no validated objective measures of wisdom or other personality traits. Finally, this was a cross-sectional study. Longitudinal investigations are necessary to link different variables in terms of temporal sequences of causal associations. A limitation of using the SDWISE-7 is that it is less suited for analysis of the seven individual subdomains of wisdom. Although each item does indicate a unique aspect of wisdom, single item indicators are expected to be less reliable measures of composite variables in comparison to multiple-item indicators (McDonald, 1999). Thus, investigators who are interested in studying subcomponents of wisdom, and not the just the broad construct, are advised to use the full 28-item version (SD-WISE-28, Thomas, et al., 2019a). Finally, while two model indices were favorable, another suggested borderline unacceptable fit for a single factor model of the SD-WISE-7. Thus, future studies should explore whether the single factor model for SD-WISE-7 total scores replicates adequately in new samples.

There is a need for large-scale multidimensional longitudinal studies of wisdom along with other measures of positive and negative well-being using biomarkers. Brief but validated measures such as the JTWI would be of practical value for such investigations. Similarly, while a number of interventions to improve components of wisdom have been shown to be effective in randomized controlled trials - i.e. wisdom is potentially modifiable (Lee et al., 2020), it is important to examine changes in scores such as JTWI along with neurobiological assessments like brain imaging.

\section{Acknowledgments}

This work was supported, in part, by the National Institute of Mental Health (K23MH119375-01 to EEL); the VA San Diego Healthcare System; and the Stein Institute for Research on Aging (Director: DVJ) at the University of California San Diego.

\section{Conflict of interest}

The authors have declared that there are no conflicts of interest in relation to the subject of this study.

\section{Description of authors' roles}

MT: Design, analysis, writing. BP: Design, critical review. EL: Design, critical review.
$\mathrm{RD}$ : Data collection/management, writing, critical review.

JL: Analysis, critical review.

XT: Analysis, critical review.

DJ: Design, analysis, writing.

\section{References}

Achenbaum, W. A. and Orwoll, L. (1991). Becoming wise: a psycho-gerontological interpretation of the book of job. The International Fournal of Aging and Human Development, 32, 21-39. DOI 10.2190/419R-X8FC-Q6NE-0M85.

Al-Refae, M., Al-Refae, A., Munroe, M., Sardella, N. A. and Ferrari, M. (2021). A self-compassion and mindfulness-based cognitive mobile intervention (Serene) for depression, anxiety, and stress: promoting adaptive emotional regulation and wisdom. Frontiers in Psychology, 12, 648087. DOI 10.3389/fpsyg.2021.648087.

Ardelt, M. (2003). Empirical assessment of a ThreeDimensional Wisdom Scale. Research on Aging, 25, 275324. DOI 10.1177/0164027503025003004.

Ardelt, M. (1997). Wisdom and life satisfaction in old age. The fournals of Gerontology Series B: Psychological Sciences and Social Sciences, 52B, P15-P27. DOI 10.1093/geronb/52B .1.P15.

Ardelt, M. and Jeste, D. V. (2016). Wisdom and hard times: the ameliorating effect of wisdom on the negative association between adverse life events and well-being. The fournals of Gerontology, Series B: Psychological Sciences and Social Sciences, 73, 1374-1383. DOI 10.1093/geronb/gbw137.

Bangen, K. J., Meeks, T. W. and Jeste, D. V. (2013). Defining and assessing wisdom: a review of the literature. The American fournal of Geriatric Psychiatry, 21, 12541266. DOI 10.1016/j.jagp.2012.11.020.

Brown, T. A. (2015). Confirmatory Factor Analysis for Applied Research (2nd ed.). New York; London: The Guilford Press.

Buhrmester, M., Kwang, T. and Gosling, S. D. (2011). Amazon's Mechanical Turk: a new source of inexpensive, yet high-quality, data? Perspectives on Psychological Science, 6, 3-5. DOI 10.1177/1745691610393980.

Campbell-Sills, L. and Stein, M. B. (2007). Psychometric analysis and refinement of the Connor-Davidson Resilience Scale (CD-RISC): validation of a 10-item measure of resilience. Fournal of Traumatic Stress, 20, 1019-1028. DOI $10.1002 /$ jts. 20271.

Cella, D. et al. (2019). PROMIS $₫$ adult health profiles: efficient short-form measures of seven health domains. Value in Health, 22, 537-544. DOI 10.1016/j.jval.2019.02.004.

Cella, D., Gershon, R., Lai, J. S. and Choi, S. (2007). The future of outcomes measurement: item banking, tailored short-forms, and computerized adaptive assessment. Quality of Life Research, 16, 133-141. DOI 10.1007/s11136007-9204-6.

Coppock, A. (2019). Generalizing from survey experiments conducted on Mechanical Turk: a replication approach. PSRM, 7, 613-628. DOI 10.1017/psrm.2018.10.

Dawes, S. E., Palmer, B. W., Allison, M. A., Ganiats, T. G. and Jeste, D. V. (2011). Social desirability does not confound reports of wellbeing or of socio-demographic 
attributes by older women. Ageing and Society, 31, 438-454. DOI 10.1017/S0144686X10001029.

de Jager Meezenbroek, E., Garssen, B., van den Berg, M., van Dierendonck, D., Visser, A. and Schaufeli, W. B. (2012). Measuring spirituality as a universal human experience: a review of spirituality questionnaires. Fournal of Religion and Health, 51, 336-354. DOI 10.1007/s10943010-9376-1.

Etezadi, S. and Pushkar, D. (2013). Why are wise people happier? An explanatory model of wisdom and emotional well-being in older adults. Fournal of Happiness Studies, 14, 929-950. DOI 10.1007/s10902-012-9362-2.

Fowler, J. H. and Christakis, N. A. (2008). Dynamic spread of happiness in a large social network: longitudinal analysis over 20 years in the Framingham Heart Study. $B M F$, 337, a2338. DOI 10.1136/bmj.a2338.

Grennan, G. et al. (2021). Cognitive and neural correlates of loneliness and wisdom during emotional bias. Cerebral Cortex, 31, 3311-3322. DOI 10.1093/cercor/bhab012.

Grossmann, I. et al. (2020). The science of wisdom in a polarized world: knowns and unknowns. Psychological Inquiry, 31, 103-133. DOI 10.1080/1047840X.2020 .1750917 .

Hauser, D. J. and Schwarz, N. (2016). Attentive Turkers: MTurk participants perform better on online attention checks than do subject pool participants. Behavior Research Methods, 48, 400-407. DOI 10.3758/s13428-015-0578-z.

Hayes, A. F. and Coutts, J. J. (2020). Use omega rather than Cronbach's alpha for estimating reliability. But.... Communication Methods and Measures, 14, 1-24. DOI 10 $.1080 / 19312458.2020 .1718629$.

Haynes, S. N., Smith, G. T. and Hunsley, J. D. (2011) Scientific Foundations of Clinical Assessment (1st ed.). New York: Routledge.

Hershey, D. A. and Farrell, A. H. (1997). Perceptions of wisdom associated with selected occupations and personality characteristics. Current Psychology, 16, 115130. DOI 10.1007/s12144-997-1019-7.

Hu, L. and Bentler, P. M. (1999). Cutoff criteria for fit indexes in covariance structure analysis: conventional criteria versus new alternatives. Structural Equation Modeling: A Multidisciplinary fournal, 6, 1-55. DOI 10.1080/ 10705519909540118.

Jason, L. A., Reichler, A., King, C., Madsen, D., Camacho, J. and Marchese, W. (2001). The measurement of wisdom: a preliminary effort. Fournal of Community Psychology, 29, 585-598. DOI 10.1002/jcop .1037 .

Jeste, D. V. and Lee, E. E. (2019). The emerging empirical science of wisdom: definition, measurement, neurobiology, longevity, and interventions. Harvard Review of Psychiatry, 27, 127-140. DOI 10.1097/HRP.0000000000000205.

Jeste, D. V., Lee, E. E., Palmer, B. W. and Treichler, E. B. H. (2020a). Moving from humanities to sciences: a new model of wisdom fortified by sciences of neurobiology, medicine, and evolution. Psychological Inquiry, 31, 134-143. DOI 10.1080/1047840X.2020.1757984.

Jeste, D. V. et al. (2020b). Study of loneliness and wisdom in 482 middle-aged and oldest-old adults: a comparison between people in Cilento. Aging $\mathcal{E}$ Mental Health, 25, 2149-2159. DOI 10.1080/13607863.2020.1821170.
Jeste, D. V. et al. (2021). Is spirituality a component of wisdom? Study of 1,786 adults using expanded San Diego Wisdom Scale (Jeste-Thomas Wisdom Index). Fournal of Psychiatric Research, 132, 174-181. DOI 10.1016/j .jpsychires.2020.09.033.

Jeste, D. V. and Vahia, I. V. (2008). Comparison of the conceptualization of wisdom in ancient Indian literature with modern views: focus on the Bhagavad Gita. Psychiatry: Interpersonal and Biological Processes, 71, 197209. DOI 10.1521/psyc.2008.71.3.197.

Koenig, H. G. (2012). Religion, spirituality, and health: the research and clinical implications. ISRN Psychiatry, 2012, 1-33. DOI 10.5402/2012/278730.

Koenig, H. G., Westlund, R. E., George, L. K., Hughes, D. C., Blazer, D. G. and Hybels, C. (1993). Abbreviating the Duke Social Support Index for use in chronically ill elderly individuals. Psychosomatics, 34, 61-69. DOI 10.1016/ S0033-3182(93)71928-3.

Kroenke, K., Spitzer, R. L. and Williams, J. B. (2001). The PHQ-9: validity of a brief depression severity measure. Fournal of General Internal Medicine, 16, 606-613. DOI 10 $.1046 / j .1525-1497.2001 .016009606 . x$.

Kroenke, K., Spitzer, R. L., Williams, J. B. W., Monahan, P. O. and Löwe, B. (2007). Anxiety disorders in primary care: prevalence, impairment, comorbidity, and detection. Annals of Internal Medicine, 146, 317-325. DOI 10.7326/0003-4819-146-5-200703060-00004.

Lee, E. E. et al. (2020). Outcomes of randomized clinical trials of interventions to enhance social, emotional, and spiritual components of wisdom: a systematic review and meta-analysis. FAMA Psychiatry, 77, 925. DOI 10.1001/ jamapsychiatry.2020.0821.

Lee, E. E. et al. (2019). High prevalence and adverse health effects of loneliness in community-dwelling adults across the lifespan: role of wisdom as a protective factor. International Psychogeriatrics, 31, 1447-1462. DOI 10.1017/ S1041610218002120.

Leeman, T. M., Knight, B. G., Fein, E. C., Winterbotham, S. and Webster, J. D. (2021). An evaluation of the factor structure of the Self-Assessed Wisdom Scale (SAWS) and the creation of the SAWS-15 as a short measure for personal wisdom. International Psychogeriatrics, 11, 1-11. DOI 10.1017/ S1041610220004202.

Li, C.-H. (2016). Confirmatory factor analysis with ordinal data: comparing robust maximum likelihood and diagonally weighted least squares. Behavior Research Methods, 48, 936-949. DOI 10.3758/s13428-015-0619-7.

Litman, L., Robinson, J. and Rosenzweig, C. (2015). The relationship between motivation, monetary compensation, and data quality among US- and India-based workers on Mechanical Turk. Behav Res, 47, 519-528. DOI 10.3758/ s13428-014-0483-x.

Mason, W. and Suri, S. (2012). Conducting behavioral research on Amazon's Mechanical Turk. Behavior Research Methods, 44, 1-23. DOI 10.3758/s13428-011-0124-6.

McDonald, R. P. (1999). Test Theory: A Unified Treatment. Mahwah, NJ: Lawrence Erlbaum Associates Publishers.

Nguyen, T. T. et al. (2020). Predictors of loneliness by age decade: study of psychological and environmental factors in 2,843 community-dwelling Americans aged 20-69 years. 
The fournal of Clinical Psychiatry, 8110.4088/JCP $.20 \mathrm{~m} 13378$.

Nguyen, T. T. et al. (2021). Association of loneliness and wisdom with gut microbial diversity and composition: an exploratory study. Frontiers in Psychiatry, 12, 648475. DOI 10.3389/fpsyt.2021.648475.

Peer, E., Vosgerau, J. and Acquisti, A. (2014). Reputation as a sufficient condition for data quality on Amazon Mechanical Turk. Behavior Research Methods, 46, 1023-1031. DOI 10.3758/s13428-013-0434-y.

Perry, C. L., Komro, K. A., Jones, R. M., Munson, K., Williams, C. L. and Jason, L. (2002). The measurement of wisdom and its relationship to adolescent substance use and problem behaviors. Fournal of Child $\mathcal{E}$ Adolescent Substance Abuse, 12, 45-63. DOI 10.1300/J029v12n01_03.

Revelle, W. (2018). psych: Procedures for Psychological, Psychometric, and Personality Research. Evanston, IL: Northwestern University.

Rizopoulos, D. (2006). ltm: an R package for latent variable modeling and item response theory analyses. Fournal of Statistical Software, 17, 1-25. DOI 10.18637/jss. v017.i05.

Rosseel, Y. (2012). lavaan: an R package for structural equation modeling. Fournal of Statistical Software, 48, 1-36.

Russel1, D., Peplau, L. A. and Ferguson, M. L. (1978). Developing a measure of loneliness. Fournal of Personality Assessment, 42, 290-294. DOI 10.1207/ s15327752jpa4203_11.

Sprouse, J. (2011). A validation of Amazon Mechanical Turk for the collection of acceptability judgments in linguistic theory. Behavior Research Methods, 43, 155-167. DOI 10 .3758/s13428-010-0039-7.

Thomas, M. L. et al. (2019a). A new scale for assessing wisdom based on common domains and a neurobiological model: the San Diego Wisdom Scale (SD-WISE). Fournal of Psychiatric Research, 108, 40-47. DOI 10.1016/j.jpsychires .2017.09.005.

Thomas, M. L., Bangen, K. J., Ardelt, M. and Jeste, D. V. (2017). Development of a 12-item abbreviated ThreeDimensional Wisdom Scale (3D-WS-12): item selection and psychometric properties. Assessment, 24, 71-82. DOI 10 $.1177 / 1073191115595714$.

Thomas, M. L. et al. (2019b). Individual differences in level of wisdom are associated with brain activation during a moral decision-making task. Brain and Behavior, 9, e01302. DOI 10.1002/brb3.1302.

Treichler, E. B. H. et al. (2020). A pragmatic trial of a group intervention in senior housing communities to increase resilience. International Psychogeriatrics, 32, 173-182. DOI 10.1017/S1041610219002096.

Ware, J. E. and Sherbourne, C. D. (1992). The MOS 36Item Short-Form Health Survey (SF-36). I. Conceptual framework and item selection. Med Care, 30, 473-483.

Webster, J. D. (2003). An exploratory analysis of a selfassessed wisdom scale. Fournal of Adult Development, 10, 13-22. DOI 10.1023/A:1020782619051.

Wink, P. and Helson, R. (1997). Practical and transcendent wisdom: their nature and some longitudinal findings. Fournal of Adult Development, 4, 1-15. DOI 10.1007/ BF02511845. 